\title{
Oral Biofilm and Its Impact on Oral Health, Psychological and Social Interaction

\author{
Gedif Meseret Abebe*
} \\ Department of Biology, College of Natural and Computational Science, Wolaita Sodo University, Ethiopia
}

*Corresponding author: Gedif Meseret Abebe, Department of Biology, College of Natural and Computational Science, Wolaita Sodo University, Wolaita Sodo, Ethiopia

\begin{abstract}
Oral health can be act as "mirror" that indicate and reflect the overall health condition, life style and oral hygiene. Oral cavity harbours arrays of microorganisms that interact with diet, oral environment, and host. This complex dynamic interaction results in microbial colonization and the subsequent oral biofilm formation. Oral biofilms are highly ordered, surface-associated assemblages of microorganisms embedded in an extracellular matrix. These surface-attached microbial communities are responsible for many oral diseases such as dental caries and periodontitis. Dental caries and periodontitis are biofilm-dependent diseases manifested within oral environment. These diseases in turn have a detrimental impact on oral health, psychology and social interaction of patients. Halitosis is a prime example of psycho-social problem resulting from oral biofilms and other health related complaints. Although oral biofilm formation is inevitable and can't be completely eliminated, the severity can be reduced through effective oral hygiene measures. Appropriate and timely hygiene procedure for the teeth and oral cavity is the most effective and specific way to prevent dental caries and periodontal diseases. Toothbrushing, interdental cleaning, and professional scaling procedures are mechanical methods required to regularly and effectively disrupt and remove the plaque biofilm. Along with oral hygiene sugary diets, smoking and alcohol consumption must be considered critically. Furthermore, preventing bacterial attachment, manipulating cell-to-cell signalling, using effective antimicrobials, and developing inhibitors that can disrupt the matrix are the promising approaches to alleviate the problems. Generally, oral biofilm is an escalating public health, psychological, and social threat that is highly linked to the food we eat, microbes we harbor and general health status. Therefore, the aim of this review article is to provide an overview of oral biofilms and its impact on health, psychological and social interactions.
\end{abstract}

\section{Keywords}

Oral health, Oral biofilm, Dental caries, Periodontitis, Psychological and social interaction

\section{Introduction}

The oral cavity is a highly dynamic environment that communicates the human body to the external environment [1]. The human oral cavity encompasses different habitats such as tongue, teeth, gingival sulcus, hard and soft palates, and tonsils [2]. It can be considered as an interface, port, and home for many microorganisms. The microorganisms found in the human oral cavity have been referred to as the oral microflora, oral microbiota, or oral microbiome [2]. Our mouth hosts a complex microbiome consisting of bacteria, archaea, protozoa, fungi, and viruses [3] and provides the appropriate space for the colonization of these microorganisms [2]. Therefore, oral cavity harbours the second most diverse microbial species [4-7], that are present in either planktonic or sessile form [8]. It is estimated that more than $95 \%$ of bacteria existing in nature are in biofilms [9]. Similarly, bacteria within the oral cavity live primarily as complex, polymicrobial biofilms [10]. Different habitats in the oral cavity allowed the existence of distinct ecological niches that promote colonization of a wide variety of microorganisms [1]. Oral cavity is an ideal place where microbes, oral fluids, nutrients, soft and hard tissues are interacting and produce unique ecosystems $[11,12]$. Therefore, the oral cavity has similar logic with that of "incubator" in which microbes can be grown based on their temperature, $\mathrm{pH}$, nutrient, and moisture preferences. This is because the warm, moist, and nutritious oral environment provides an ideal hatchery for microbial growth and proliferation [13].

From a medical point of view, oral health is a key indicator of overall health, well-being and quality of life [14]. It can act as a "mirror" that indicates and reflect

Citation: Abebe GM (2021) Oral Biofilm and Its Impact on Oral Health, Psychological and Social Interaction. Int J Oral Dent Health 7:127. doi.org/10.23937/2469-5734/1510127

Accepted: March 10, 2021: Published: March 12, 2021

Copyright: @ 2021 Abebe GM. This is an open-access article distributed under the terms of the Creative Commons Attribution License, which permits unrestricted use, distribution, and reproduction in any medium, provided the original author and source are credited. 
the overall health condition, lifestyle and oral hygiene. A disease that is localized elsewhere in the body can be reflected in the mouth [15]. The bacteria colonizing the hard and soft tissues of the oral cavity are known to significantly influence oral health and causing diseases $[5,16]$. Oral microbes attached to hard and soft tissues can produce biofilms which are highly ordered, surface-associated assemblages of microbes embedded in an extracellular matrix $[17,18]$. This surface-attached microbial community is responsible for many oral diseases. Major disturbances in the interactions between oral microbes, the microenvironment, and the host may lead to the development of oral diseases [1]. For instance, the dynamic balance between commensals and opportunistic pathogens can be disrupted by frequent sugar consumption and poor oral care, which promotes the development of virulent biofilms in close proximity to the tooth surface [19]. As Jiao, et al. reported, the complex dynamic interactions among microorganisms, hosts, and diet result in microbial colonization and the subsequent formation of pathogenic biofilms [13]. The nature of surfaces in which microbes can be attached play a critical role in biofilm formation. For instance, the eruption of teeth provides a unique, hard non-shedding surface that enables much larger masses of microorganisms (dental plaque) to accumulate as biofilms [15]. Teeth are the hardest tissues [20] that are used as a substratum for microbial attachment and biofilm formation. Biofilm formation on teeth surfaces has similar logic with the growth of alga on rock surfaces. Thus, saliva irrigated microbes produce a conspicuous tar-like biofilm (dental plaque) on teeth surface which is very difficult for eradication. Since the oral cavity act as a port of entry and a "mini bioreactor", nutrients such as sugars are changed into different microbial metabolic products such as acids and glue-like extracellular polymeric (EPS) substances that are important for biofilm formation and evolution of dental caries (Figure 1 and Figure 2). If dental plaque is not removed frequently at its infant stage, then the biofilm undergoes maturation, and the resulting pathogenic bacterial complex can lead to dental caries, gingivitis, and periodontitis [21]. Today, dental caries is regarded as a preventable non-communicable disease (NCD) that affects a majority of the population across their lifespan [22]. Dental caries is a biofilm-induced disease, resulting from interactions of microorganisms, host-related factors, and diet (sugars), which modulate the dynamic formation of biofilms on tooth surfaces $[23,24]$. Therefore, oral biofilm is one of the virulence factors of oral infectious diseases such as dental caries, gingivitis, periodontitis, periapical periodontitis, and peri-implantitis [18]. Our oral cavity and its environment take its own shares in giving beauty and self-esteem for us. However, any defect in this environment such as the manifestation of dental caries, periodontal disease, tooth loss, and malodor have a detrimental impact on health, psychology, and social interactions of an individual [14]. As general the unique conditions in the oral cavity including moisture, temperature variations, presence of hard tissues, carbon, and nitrogen, make this a suitable environment for many microbial species $[20,25]$ and it is an ideal place for oral biofilm formation [25], which has a detrimental impact on health, psychology, and social interactions. Even if oral biofilms are widely studied as model systems for bacterial adhesion, biofilm development, and biofilm resistance to antibiotics, still its formation within this cavity is inevitable and it is escalating health, psychological, and social-interaction threat that is highly linked to the food we eat, microbes we harbor and our general health status. Hence, the aim of this review article is to provide an overview of oral biofilms and its impact on health, psychological, and social interactions.

\section{Stages of Oral Biofilm Formation}

In the annals of microbiology and biofilm, Antonie Van Leeuwenhoek is always remembered by his tremendous contribution to the realization and development of these fields. He tried to observe plaque off his own teeth under his primitive light microscope [10] and observed microorganisms that dart back and forth before the movement of his eyes and finally he called them as animalcules, meaning little animals in that period. Since then, his experiment and discovery are a milestone in oral biofilms and paved ways towards more understanding of microbial biofilms. Biofilm formation on different biotic or abiotic surfaces is a dynamic and stepwise process culminated from bacterial, surface and nutrient-related factors and interactions. As Donlan and Costerton reported whatever ecosystem they inhabit bacteria produce biofilm in a similar way or fashion [26]. As shown in Figure 1 oral biofilms are physiologically and structurally organized groups of microbial communities that are embedded in an extracellular matrix of exopolymers on mucosal and dental surfaces [27]. The life of an oral biofilm may be depicted as a developmental cycle (Figure 1). Therefore, biofilm formation within oral cavity is cyclical stages which require: a) Reversible attachment of planktonic bacteria to conditioned solid surfaces such as teeth surfaces; b) Synthesis of glue-like exopolysaccharide matrix; c) Cells are firmly attached to the surface which is irreversible; d) Production of matured biofilm structure; e) Dispersion of an organized structure, and f) Seeking new habitats [28] (Figure 1). These stages of the cycle are determined by physical, biological and environmental factors. Generally, the formation of dental biofilm (or dental plaque) consists of several steps, which start with the formation of the acquired enamel pellicle, followed by the initial adhesion of planktonic bacteria to the pellicle layer by binding sites, subsequent maturation of the bacterial biofilm and, finally, the dispersion of biofilm with detachment of cells/clusters of cells [29] (Figure 1).

The acquired enamel pellicle (AEP) is a protein 
film with unique composition and properties, which is formed by the selective adsorption of a variety of oral fluid-derived proteins onto tooth enamel surfaces [30]. Therefore, the composition of the acquired pellicle can modify the mineral homeostasis of the tooth surfaces and the attachment of bacteria for the development of the biofilm [31]. The formation of acquired pellicle is the first step in dental biofilm formation and it distinguishes dental biofilms from other biofilm types [29] (Figure 1). The salivary pellicle that coats the tooth surfaces is mediating the attachment of oral bacteria on to these surfaces [32]. This is the critical step that makes teeth surfaces conducive for attachments of initial colonizers and this again opens a door for attachment of the second species. In this fashion, they develop mature three-dimensional structure i.e. oral biofilm that can be seen on teeth surfaces as dental plaque. Majority of initial colonizers of teeth are Gram-positive facultative anaerobic cocci and rods, including Streptococcus and Actinomyces species. These initial colonizers provide a foundation for further development of dental biofilm [12] (Figure 1). Therefore, the presence of one microorganism creates the ecological niches for other microorganisms, which facilitates their survival in the new favorable conditions [28]. Along with attachment and proliferation microbes produce different metabolic products such as acids on teeth surfaces (Figure 1 and Figure 2). Acids produced in oral cavity can be considered as an "excavator" that can dig and produce "caves" on the surfaces of teeth. In dental caries, acidogenic enamel-attached bacteria like streptococci metabolize dietary carbohydrates to organic acids [33]. Low pH at the tooth surface leads to enamel demineralization, then enamel cavities which can progress into the tooth dentin $[17,33,34]$. Therefore, the frequent acidification selects for more aciduric species, like Streptococcus mutans, that further lower the $\mathrm{pH}$ [33]. The consistent presence of acid in the environment disrupts the mineral equilibrium of the exposed dental structures (i.e. enamel and/or dentin), and, therefore, leads to carious lesions [29]. Acidic environment also imposes environmental stress on the microbial community causing acid-sensitive species to perish and aciduric microbiota to flourish [35].

Figure 1 Schematic of the bacterial biofilm formation process on teeth surfaces: The development process of biofilms involves the initial deposition of microbial cells onto a conditioned tooth surface (pellicle), reversible attachment to the substratum, irreversible attachment and colonization, aggregation and expansion, biofilm maturation, and finally biofilm dispersal and controlled detachment $[15,36]$.

As shown in Figure 1 microbe are secreting cement-like extracellular polymeric substance (EPS) that are important for attachment [10] (Figure 1). The EPS acts as biological glue that enhances the adherence of

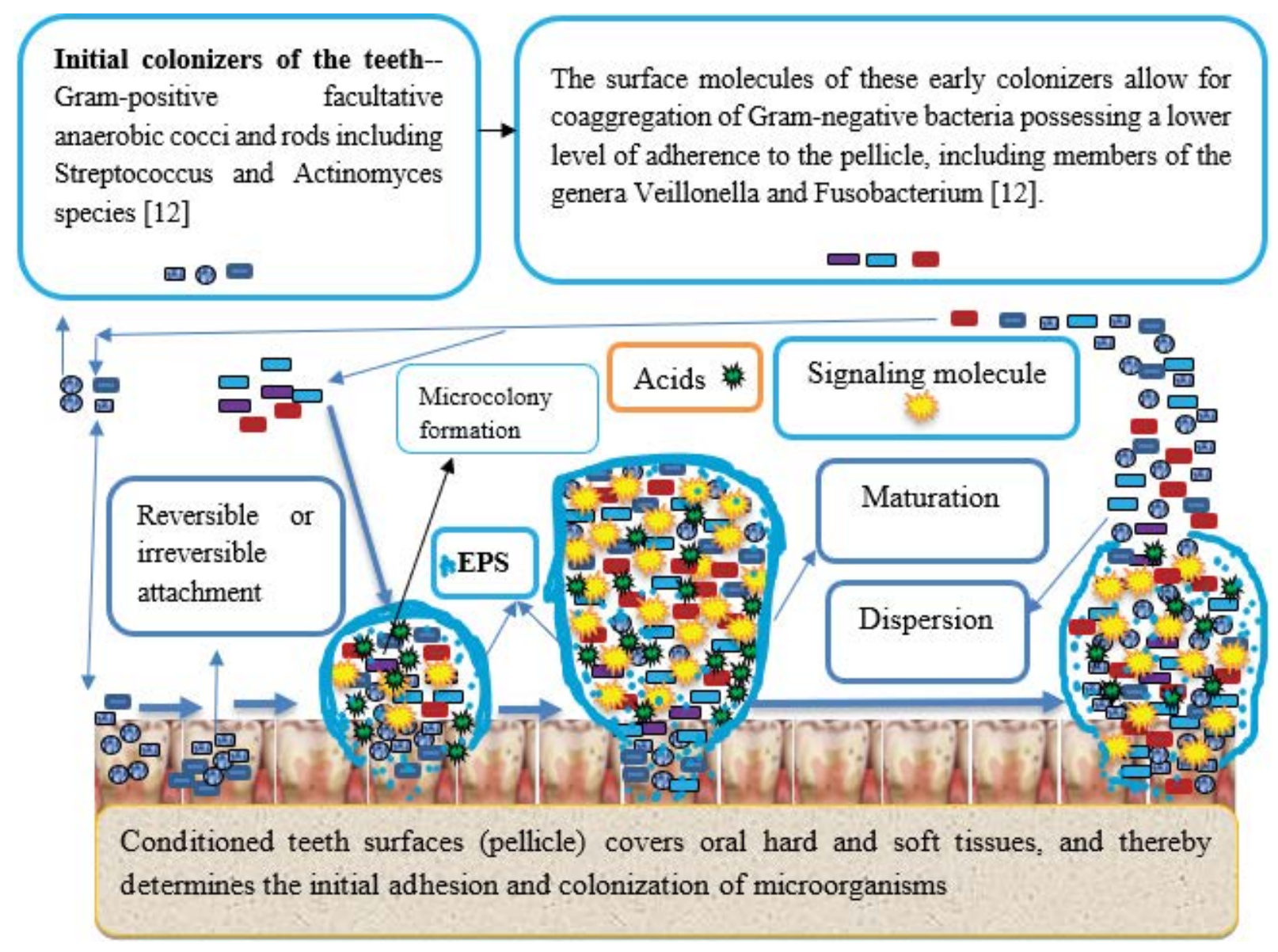

Figure 1: Schematic of the bacterial biofilm formation process on teeth surfaces. 


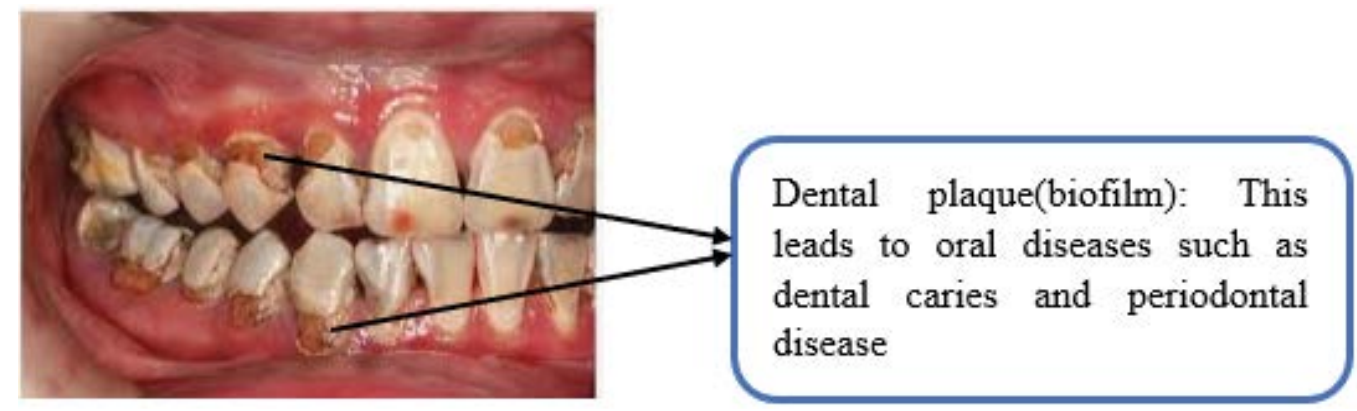

Figure 2: Dental plaque formation.

microorganisms to each other and to the tooth surface, which increases dental biofilm formation [37]. This matrix helps to protect bacteria within the biofilm from antibiotics, antiseptics and host immune system [21]. EPS production directly mediates microbial adherence to a surface and cell-to-cell adhesion, while forming a polymeric matrix that enhances mechanical stability of biofilms [19]. Additionally, EPS is able to enhance the diffusion pattern of acids throughout the biofilm matrix by increasing the porosity of the extracellular matrix [37]. The tar-like dental plaque that can be found on teeth surface are recalcitrant to sanitation or cleansing due to such kinds glue-like materials. The good evidence for this notion is checking your own teeth if there is tar-like plaque and then try to remove it, unfortunately they are recalcitrant and will not be removed using mechanical methods such as toothbrushing (Figure 2). Moreover, the EPS matrix keeps the bacteria banded together, in order that they aren't flushed away by the action of saliva and gingival crevicular fluid [21]. The EPS matrix not only used for attachment but also creates a unique microenvironment that is different from other sites in terms of key environmental growth factors $\mathrm{pH}$, redox, oxygen, and nutrient availability [13]. Microbial cells within a biofilm are very close to each other so that they can communicate through chemicals which enable them to coordinate and respond to any ecological, environmental, and host-related cues [38]. As shown in Figure 1 microorganisms are in close proximity to one another in biofilms, thereby providing many opportunities for synergistic interactions [39]. This cell-to-cell proximity and interaction are important for communication (Quorum sensing), horizontal gene transfer, and division of labor.

For example, bacteria can cooperate with one another to metabolize complex host molecules, such as glycoproteins, that would be recalcitrant to the action of single species [39]. As shown in Figure 1 bacterial cells are able to communicate with one another in biofilm communities via small diffusible molecules (yellow-colored molecule) [40]. In order to control, coordinate, and manage communal activities bacterial cells use density-dependent signaling molecules as a "language". Therefore, bacteria in dental plaque communicate with each other through a variety of chemical signaling mol- ecules [35]. To sum up, bacterial inter-communication is a main process supporting colonization and biofilm formation on the dental enamel [20] (Figure 1).

Figure 2 Dental plaque formation: Dental plaque is a sticky film comprising multiple bacteria assembled as biofilm on surface orperiphery of teeth. It consists of highly structured complex that allows sequential bacte$\mathrm{rial} / \mathrm{microbial}$ succession [16]. Teeth allow the accumulation of large masses of microorganisms (predominantly bacteria) and their extracellular products, termed dental plaque [15] (Figure 2). As shown in this figure the formation of such kinds of dental plaque leads to oral diseases such as dental caries and periodontal diseases.

\section{Factors that Determine Bacterial Growth and Oral Biofilm Formation}

Oral bacterial communities may be influenced by physical and biological factors, nutrient availability, $\mathrm{pH}$ and oxy-reduction potential, as well as diet, smoking and drinking habits and the patient's overall health and oral hygiene. These determinants may change bacterial growth and the biofilm characteristics [41,42]. Even if all these factors are crucial in determining the bacterial growth and biofilm formation only few are discussed here below (Figure 3).

\section{Saliva}

Saliva is one of the critical components of oral environment that irrigate our mouth and the teeth surface to produce conditioned films for microbial adhesion and biofilm formation. As Marsh, et al. noted saliva plays a tremendous in determining the composition and activity of oral microbes through different mechanisms such as forming conditioning film on oral surfaces, buffers the $\mathrm{pH}$ in the biofilm to around neutrality, and delivers components of the adaptive and innate host defenses [15]. The nature of surfaces and the presence of a conditioning film (pellicle) are important factors in the microbial attachment to a solid surface such as teeth surfaces [26]. Therefore, saliva contributes to the formation of the salivary pellicle, which covers the oral hard and soft tissues, and thereby determines the initial adhesion and colonization of microorganisms [23]. Therefore, saliva plays an important role in controlling the growth of biofilm [21]. Numerous compounds are dissolved in saliva 


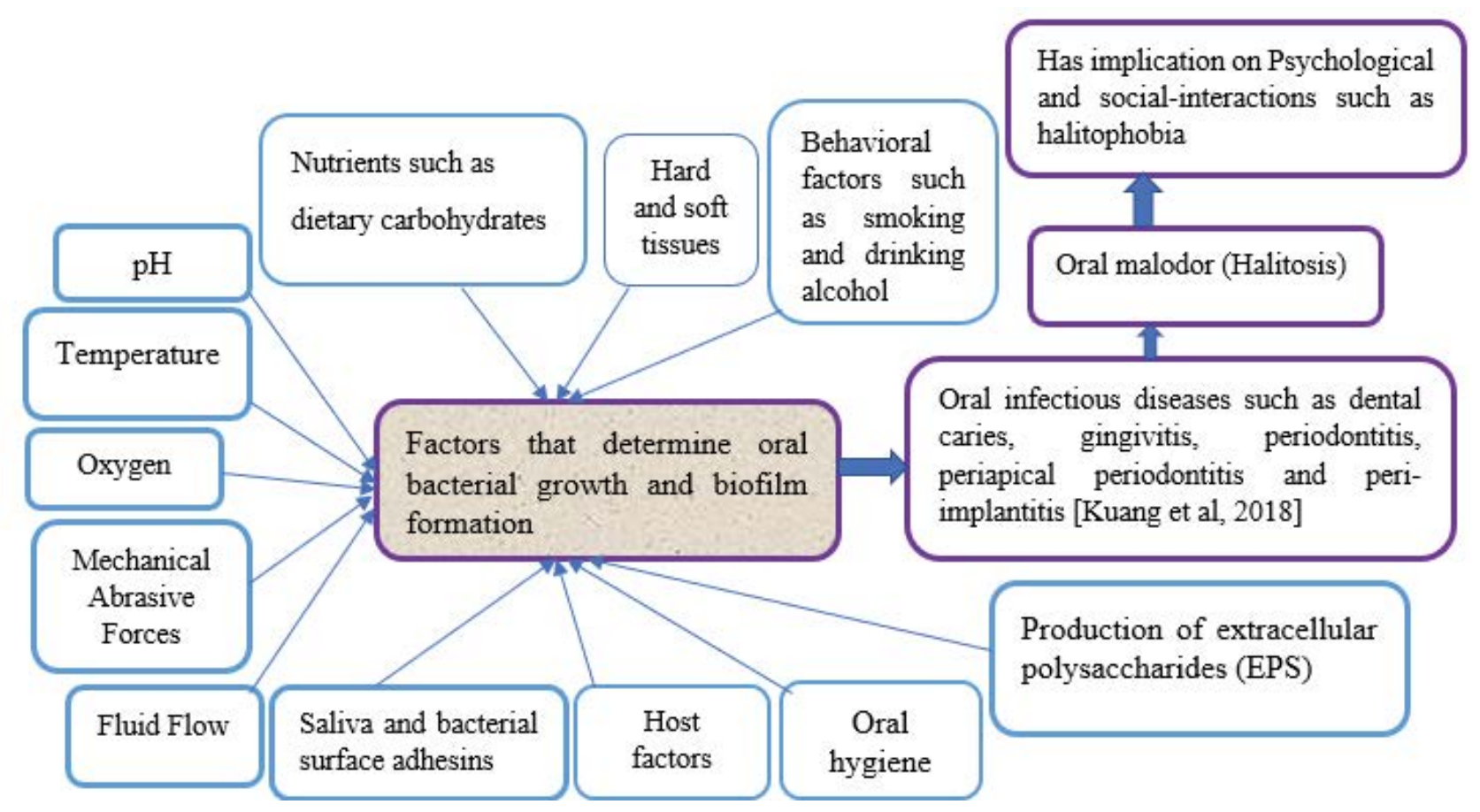

Figure 3: Factors that determine bacterial growth and oral biofilm formation and its implication on oral health, psychology, and social interactions and the interaction $[15,28]$ of these factors with oral microorganisms may lead to deleterious relationship that trigger oral infectious disease and this in turn may lead to psychological and social-interaction complaints.

and may influence the composition of oral biofilms and bacterial growth [33]. Saliva not only helps to maintain an environment that allows biofilms to flourish, but also modulates the layers of plaque with the help of numerous proteins, including enzymes and glycoproteins, and minerals, which control biofilm build-up and activity [6].

\section{Nutrient}

Dental diseases result from a complex interaction of the environment, the resident microbes, and the host [15]. Nutrients found inside our mouth along with other components play a critical role in oral biofilm formation. Dietary sugars can change the structure and composition of biofilms formed on teeth surfaces and the residing microbial communities become highly fit to metabolize carbohydrates and produce acids leading to dental caries $[19,43]$. A person who regularly consumes foods with a high fermentable sugar content has greater proportions of streptococci mutans and lactobacilli in plaque [44]. Oral biofilm formation on teeth surface is a spot point for the formation of dental caries. Dental caries is a biofilm-dependent disease associated with frequent intake of a diet rich in rapidly fermented carbohydrates that are converted to acidic end-products by biofilm microbiota [37]. Among the carbohydrates, sucrose is considered the most cariogenic, since it is a substrate for the synthesis of extracellular (EPS) and intracellular (IPS) polysaccharides [45]. Therefore, sucrose is the most cariogenic sugar because it can form glucan that enables firm bacterial adhesion to teeth [46]. Dental caries cannot develop without the presence of dietary fermentable carbohydrates, in particular sug- ar [47]. Dental caries in preschool children is high due to a combination of factors, such as the colonization of teeth with cariogenic bacteria, type of foods, and frequency of exposure of these foods to the cariogenic bacteria, and susceptible teeth [46]. Generally, dietary sugars play a critical role in oral biofilm formation and evolution of oral diseases such as dental caries.

\section{Oral hygiene}

As previously mentioned, oral health is a key indicator of overall health status, oral hygiene, and lifestyle. The oral cavity is the main gates of food, microorganisms, and home for both commensal and pathogenic microbes. Therefore, maintaining oral hygiene is an essential prerequisite for all community members. If we are unable to maintain oral hygiene, then oral diseases and conditions will be manifested and this in turn has health, psychological, social, and economic implications. For instance, poor oral hygiene can lead to serious diseases, such as periodontitis, tooth decay, pain and discomfort in teeth or gums, infections, and loss of teeth [48]. Poor oral hygiene is a foundation for biofilm formation on both soft and hard tissues. If surface attached pellicles, food particles, sugars, and other debris are left uncleaned, then oral microbes will use this opportunity to build their "house" (dental plaque) which is recalcitrant to removal and cleansing. Once they develop tar-like dental plaque (biofilm) on the surface of teeth, then disrupting this structure will be a challenge and leads us to visit the dentist, or we may lose our teeth once and for all if it is left untreated. Poor oral hygiene is the cause of biofilm accumulation and that a relatively specific group of indigenous oral bacteria is 
the cause of plaque-induced inflammatory periodontal diseases [48]. Therefore, there must be a continuous follow-up and check-up and maintaining oral hygiene. Oral hygiene maintenance is the cheapest form of preventive health measure. However, it is the most ignored practice in the underprivileged rural communities [49]. Lack of awareness, poor educational level, and poorer knowledge of oral hygiene are the most likely reasons for the ignorance of this practice. Besides this suggested reasons, lack of commitment is one of the most critical problems related to poor oral hygiene. In addition to this, adequate dental and oral hygiene may become a challenge for all users and especially for elderly people and young children because of their limited motor skills [50]. As Qin, et al. reported, a lower maternal education level, poor knowledge of oral hygiene, night-feeding, and excessive sugar intake were important contributors to the development of dental caries in children [51]. Similarly, Wulaerhan, et al. reported that caries prevalence was associated with oral hygiene behaviors of children and the general oral health knowledge of caregivers [52]. Night feeding and sleeping without cleaning our mouth are just like putting inoculated medium into an incubator. Any mechanical and fluid mobility that disrupt microbial activity is reduced during sleeping. As studies revealed night-feeding and eating sweets several times a day were significantly more common in severe early childhood caries (S-ECC) children than in caries-free children [51]. Therefore, dental caries risk is greatest if sugars are consumed at high frequency and are in a form that is retained in the mouth for long periods without cleaning [46] When we are sleeping, oral microorganisms are active and doing their activity. That is why in the morning our teeth and tongue surfaces are coated with oral debris and microorganisms along with malodor. Poor oral hygiene not only is closely linked to various oral health problems but also has a significant effect on oral malodor. For instance, uncleaned tongue usually harbours periodontal bacteria such as Prevotella intermedius, Porphyromonas gingivalis, and Fusobacterium species that are responsible for producing volatile sulfur compounds (VSC) that account for halitosis [53]. Generally, poor oral hygiene, sugary foods, lifestyle, oral microbes, and host-related factors determine the fate of oral biofilm formation and this in turn induces the evolution of oral diseases.

\section{The Impact of Oral Biofilm on Health}

Biofilm is a surfaced-attached microbial community that significantly affect oral health. For instance, periodontitis, caries, and peri-implant disease are infectious diseases of the oral cavity in which oral biofilms play a causative role $[17,54,55]$. These infections induced by oral biofilm may influence the quality of life, systemic health, and expenditure [17]. Synergy and interaction of variable oral microorganisms help human body against invasion of undesirable stimulation outside. However, imbalance of microbial flora contributes to oral diseas- es and systemic diseases [2]. According to the World Health Organization report around 3.56 billion people worldwide were affected by oral diseases in 2016, with untreated dental caries being among the most prevalent non-communicable diseases [14]. Besides its impact on health, it is also a major economic problem. In some countries, oral diseases are the fourth most expensive diseases to treat [56].

Teeth are colonized by oral bacteria from saliva containing more than 700 different bacterial species. Under relatively stable conditions, a complex biofilm containing up to 100 bacterial species at a site will build up and may eventually cause the development of diseases such as dental caries and periodontal diseases [4]. Microorganisms that cause dental caries and periodontal diseases are metabolically highly specialized and organized as multispecies microbial biofilms [57]. Oral bacterial biofilms trigger chronic inflammatory responses in the host that can result in the tissue destructive events of periodontitis [58]. Biofilm-induced periodontal diseases comprise a wide range of inflammatory conditions that affect the supporting structures of the teeth (the gingiva, bone, and periodontal ligament), which could lead to tooth loss and contribute to systemic inflammation [43]. These diseases are highly prevalent and can affect up to $90 \%$ of the worldwide population. Periodontal diseases frequently occur in human mouth, and can be divided into two categories, gingival diseases and periodontitis [2]. Gingivitis, the mildest form of periodontal disease, is caused by the bacterial biofilm (dental plaque) that accumulates on teeth adjacent to the gingiva (gums) [44]. The other disease manifested in oral cavity is dental caries which is one of the most common chronic and multifactorial diseases affecting the human population [59]. Dental caries has a wide range and high incidence, which can occur at any age, from children to the old [2]. Dental caries remains a significant public health problem in most developed countries, affecting $60-90 \%$ of schoolchildren and the vast majority of adults [60]. Dental caries is one of the most prevalent in children across the globe [61]. As Cirino and Scantlebury reported the rate of dental caries in developing countries is also rising [62]. Similarly, Miura, et al. reported that the prevalence of dental caries in developing countries increases with the degree of urbanization [63]. Poor oral hygiene is the possible contributing factors for the increment of dental caries in children. Dental caries result from a dysbiosis of tooth-associated biofilms [34]. Dental caries is a biofilm-induced, sugar-driven, multifactorial, oral disease that results in the demineralization of dental hard tissues $[45,64]$. In the initiation and development of dental caries, the role of fermentable dietary carbohydrates is paramount. Increasing consumption of sugary foods in the developing countries, poor tooth brushing habits, and low level of awareness are some of the factors that increased the levels of dental caries [65]. Nowadays sugar consumption is increasing both in 
developing and developed nations which has detrimental to oral health and leads to dental problems. A study conducted in Dewa Chefe District; northeast Ethiopia indicated almost all (98\%) of children consumed sugar fortnightly [66]. For instance, in the rural areas of West Gojjam, Ethiopia when mothers are going to market, they will buy candies and other sugar confectionaries to their children. Even candies and sugar confectionaries are frequently given to children when they are disturbing, crying, or as an incentive to help their parents. This gift given by their mother has a detrimental impact on their teeth and oral health as a general. Since there is poor brushing habit, sugars and other food particles can linger on teeth surfaces and in hard-to-reach spots that lead proliferation and biofilm formation and in turn microbes can produce acids and "glue" like materials which can facilitate the production of dental caries. Therefore, dental caries is a severe problem in such areas and age groups. Although children are the highly susceptible age groups, this problem is also an issue of all age groups who lives both in urban and cities. As Tafere, et al. noted lifestyle, hygiene, eating habits, inadequate fluoride, social status and sociodemographic factors contribute to the evolution of dental caries [65]. Most oral diseases and conditions share modifiable risk factors such as tobacco use, alcohol consumption, and unhealthy diets high in free sugars, all of which are increasing at the global level [14]. Therefore, socio-economic and behavioral factors play an important role in the etiology of the disease [59]. Bacterial biofilms on the tooth surface play a key role in the pathogenesis of periodontal diseases, endodontic infections, caries, and numerous other conditions extending beyond the oral cavity [36]. Generally, dental caries and periodontal diseases are not the only oral diseases caused by oral biofilms, but there are also others such as, mucosal diseases, peri-implantitis, and oral cancer Again this oral microbial dysbiosis is linked to oral inflammation and may contribute to systemic conditions through bacteremia [2]. Although there is progress in the oral health of the population globally, problems still persist particularly among poor and disadvantaged groups in both developed and developing countries [60].

\section{Psychological Effects of Oral Biofilms}

Oral health affects people physically and psychologically and influences their life, look, speech, mastication, food tasting, and feelings of social well-being [56]. The mouth plays a critical role in speech or facial expressions and makes a significant contribution to our appearance. Therefore, maintaining a healthy mouth is important for a person's self-esteem and general health [15]. Diseases and conditions such as dental caries, periodontal disease, and tooth loss manifested in our oral environment [14] have a psychological and social impact on patients. Individuals having such kinds of defects on their mouth may not be as confident as others who have normal health conditions. For instance, those individuals who possess the above-mentioned health defects may refrain from speaking, talking or other social interactions that reveal their health problems. Dental caries can affect mastication function, speech, smile, and psychosocial environment, and the quality of life of the child and the family [61]. As indicated in Figure 2, a person having such kinds of teeth may not be comfortable in front of their colleagues even to laugh or smiles like that of others who have a healthy oral environment and teeth. Oral biofilm is a critical public health issue that has a pivotal role in health, social interaction, and psychology. Pathogenic microbes residing in the oral cavity have the power to disrupt our beauty, psychology, social interaction, friendship, confidence, love, teeth, and even fresh air circulating within the cavity and leads into more health complaints (Figure 2). Teeth surface is not the only structure affected by oral biofilm, but also the air circulating inside the mouth. This paves a way for oral malodor which leads to psycho-social problems that offense both the patient and others who are around there. Oral malodor is attributed to oral microbes that affect both the psychological and social life of individuals and is the main reason to visit the dentist [67]. Hence, biofilm formation within the oral cavity is not only the issue of health; it is also a psychological and social issue within our community. Halitosis is a good example that supports this notion which is a worldwide problem that leads psychosocial and social stigma [68], and those individuals having this problem might be disturbed and refrain from group interaction. Halitosis affects the social interactions of people in daily life by causing personal discomfort and emotional stress [53]. Halitosis has a deleterious impact on patients that leads to halitophobia. Halitophobia is a fear that the patient's breath will be regarded as a bad smell by other people. In halitophobia, the patient is worried about having continuous oral malodor [67]. Therefore, halitosis is a common symptom mainly caused by microbial activities (biofilm formation) in the oral cavity such as teeth and tongue surfaces. The tongue is one of the primary sources of halitosis [69]. This is because the papillary structure of the dorsum of the tongue provides refuge for many microorganisms which would otherwise be removed by mastication and salivary flow [15]. Oral biofilm plays a tremendous role in causing infectious diseases such as periodontitis and caries in the oral cavity $[10,54]$ and also linked to disease outside oral cavity [10]. These oral diseases adversely affect children and other age groups physically and psychologically and increase the economic burden on families and society [70]. Generally, oral biofilm is escalating public health, psychological, and a social threat that is highly linked to the food we eat, microbes we harbor and general health status.

\section{Prevention}

The role of oral biofilm in causing oral disease is par- 


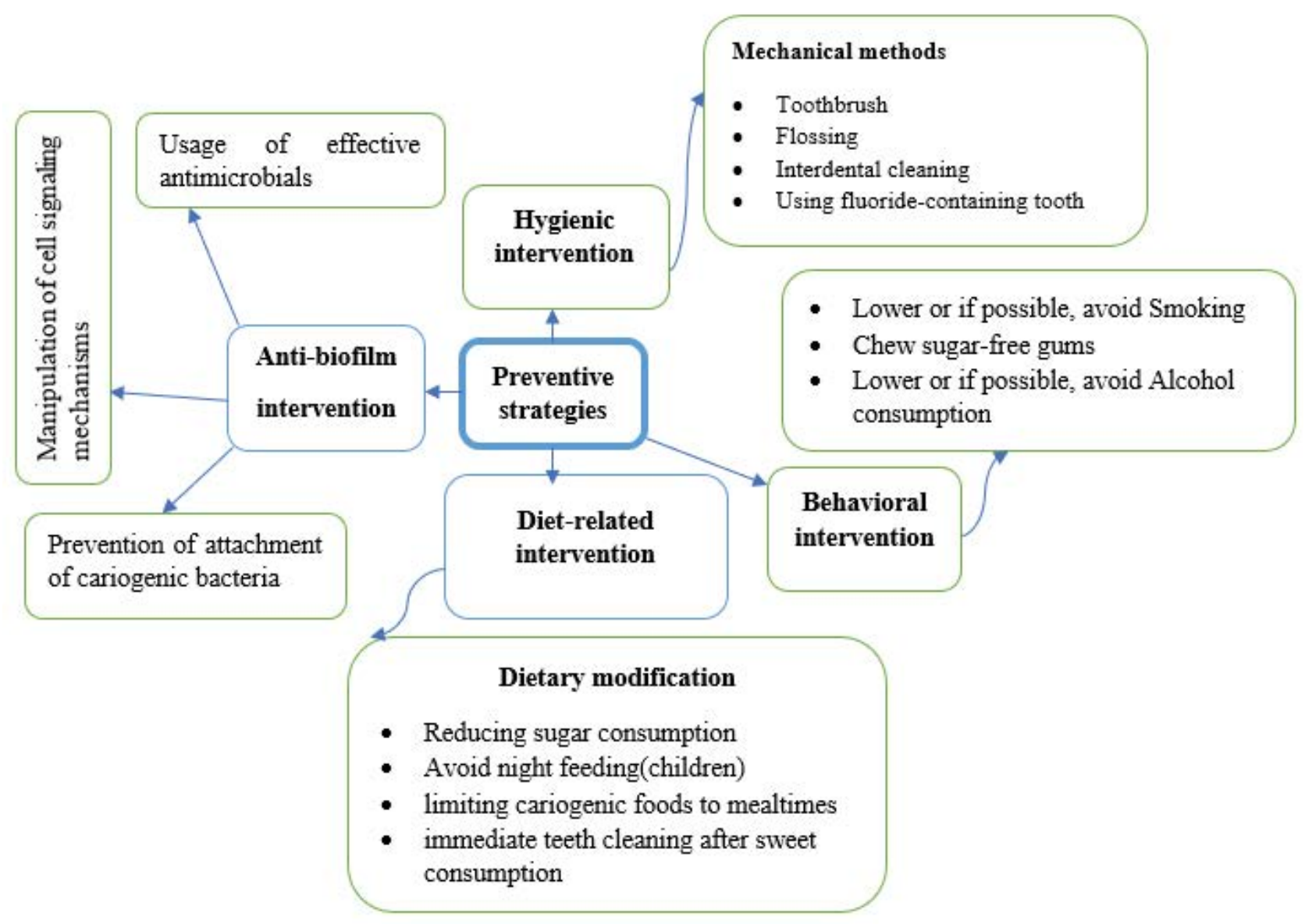

Figure 4: Dental caries preventive strategies which encompass hygienic, behavioral and antibiofilm interventions $[13,21,42,44]$.

amount. Unless biofilms are appropriately controlled, they accelerate their physiological heterogeneity and a series of complex interactions and cause tooth mineralization and systemic inflammation [42]. Most of oral diseases manifested within this cavity are not self-limiting and without adequate intervention, the process can continue until the tooth is destroyed [24]. For instance, if we are too late to prevent dental caries at its infant stage, we may lose our teeth once and for all. Dental plaque accumulates preferentially at stagnant sites that afford protection from the vigorous removal forces that are applied in the mouth [40]. The arrangement and structure of teeth provide such protection and some sites are acting as a cave that can hide microorganisms from any hostile conditions such as toothpaste, brushing, and other mechanical methods and makes the intervention measures to be ineffective. Due to this reason, effective control of oral biofilm infectious diseases represents a major global challenge [13]. Although dental biofilm cannot be completely eliminated, its pathogenicity can be lessened through effective oral hygiene measures [21].

Since oral diseases are a multifactorial problem, it is not solved using single intervention approaches. For instance, dental caries is a multifactorial disorder and it is difficult to assess all the associated risk factors simultaneously [24]. Therefore, when preventive ap- proaches are designed it is better to consider different contributing factors such as diet, behavior, oral hygiene, knowledge, age, and other health-related factors. Most oral diseases and conditions manifested in the oral cavity are biofilm-induced. Therefore, prevention of such disease is achieved with daily self-performed oral hygiene and professional removal of the microbial biofilm on a quarterly or bi-annual basis [43]. Prevention and treatment are targets to control bacterial biofilm and other risk factors and restoring lost tooth support [44]. Strategies that can disrupt any stage (e.g. microbial attachment, biofilm maturation, and biofilm dispersal) of biofilm formation are considered potentially valuable in controlling biofilm-related infections [13] (Figure 4). These preventive strategies include the prevention of attachment of cariogenic bacteria, manipulation of cell signaling mechanisms, and using effective antimicrobials, etc. [21,44] (Figure 4). Researchers also suggested in developing inhibitors that can disrupt the stability of the 3D matrix is one of a promising direction in preventing oral biofilm and its associated risk factors. Toothbrushing, interdental cleaning, and professional scaling procedures are mechanical methods required to regularly and effectively disrupt and remove the plaque biofilm [21]. Tooth brushing and flossing are fundamental for the control of dental biofilms because they can be applied without surgical intervention. Good 
oral hygiene, appropriate use of fluorides, avoiding frequent consumption sugar-containing drinks, avoid night feeding of children; promoting non-cariogenic foods for snacks, limiting cariogenic foods to mealtimes, and restricting sugar-containing snacks that are slowly eaten (e.g., candy, cough drops, lollipops, suckers) can help in preventing this disease in children $[46,61]$ and some of these preventive approaches are also applicable for other age groups. The daily use of fluoride toothpaste is seen as the main reason for the overall decline of caries worldwide over recent decades [64]. However, getting this toothpaste in underprivileged rural communities is difficult. Therefore, it is better to aware of them to maintain their oral hygiene using locally available tooth brushing methods. Apart from brushing, the complete concept of oral hygiene like frequency and duration of brushing; time of brushing; flossing; and so on should be considered and improved among individuals of different age groups and also in different parts of the world [70]. As Santos explained most adults brush and floss inadequately, and constant education and/or reinforcement is often required [71]. Most children do not remove dental caries using mechanical methods effectively which means the deficiency of maintenance of good oral hygiene [24]. Thus, oral hygiene procedures require constant guidance throughout life's different Furthermore, elimination of risk factors such as smoking, and alcohol consumption can reduce oral diseases [72].

\section{Conclusion}

The oral cavity is a highly dynamic environment that communicates the human body to the external environment. Within the oral cavity, there is a complex interaction between oral microorganisms, diet, oral environment, and host. This complex dynamic interaction results in microbial colonization and the subsequent oral biofilm formation which is a highly structured surface-attached microbial community. Dental plaque is a prime example of oral biofilm which is produced on the surfaces of teeth. Oral biofilm is responsible for the most frequently occurring oral diseases, such as dental caries and periodontal diseases. Dental caries is a complex dieto-bacterial disease with an association of social, behavioral, and biological factors. Therefore, there are a number of contributing factors to the evolution of such oral diseases. Sugary diets, oral pH, poor oral hygiene, smoking, and alcohol consumption are the forefront contributing factors for the evolution of such oral diseases. Since oral diseases are multifactorial, tracing the contributing factor must be top priority to mitigate the problem. In order to prevent the development of oral biofilm and associated risk factors, different methods have been suggested. This includes mechanical methods such as toothbrushing, flossing, interdental cleaning, and professional scaling procedures. Along with the above-mentioned mechanical approaches good oral hygiene and limiting sugary foods or frequent cleaning after consumption are the main approaches that can al- leviate the problem. The last but not the least is related to smoking and alcohol consumption. Here personal commitment is needed to save your teeth and maintain oral health. Although oral diseases are the issue of all age groups, children and elders need especial attention.

\section{Data Availability}

The data used to support the findings of this study are available from the corresponding author upon request.

\section{Conflicts of Interest}

I declare that there are no conflicts of interest in regard to the publication of this paper.

\section{Acknowledgments}

The author would like to thank Wolaita Sodo University but no fund is taken to conduct this review article.

\section{References}

1. Colombo APV, Souto RMD, Silva Boghossian CMD, Miranda R, Baêta Lourenço TGM (2015) Microbiology of oral biofilm-dependent diseases: Have we made significant progress to understand and treat these diseases? Curr Oral Health Rep 2: 37-47.

2. Gao L, Xu T, Huang G, Jiang S, Gu Y, et al. (2018) Oral microbiomes: More and more importance in oral cavity and whole body. Protein Cell 9: 488-500.

3. Mosaddad SA, Tahmasebi E, Yazdanian A, Mohammad BR, Alexander S, et al. (2019) Oral microbial biofilms: An update. Eur J Clin Microbiol Infect Dis 38: 2005-2019.

4. Roberts AP, Mullany P (2010) Oral biofilms: A Reservoir of transferable, bacterial, antimicrobial resistance. Expert Rev Anti Infect Ther 8: 1441-1450.

5. Larsen T, Fiehn NE (2017) Dental biofilm infections-an update. APMIS 125: 376-384.

6. Kilian M, Chapple ILC, Hannig M, Marsh PD, Meuric V, et al. (2016) The oral microbiome-an update for oral healthcare professionals, Br Dent J 221: 657-666.

7. Deo PN, Deshmukh R (2019) Oral microbiome: Unveiling the fundamentals, J Oral Maxillofac Pathol 23: 122-128.

8. Berger D, Rakhamimova A, Pollack A, Loewy Z (2018) Oral biofilms: Development, control, and analysis. High-Throughput 7.

9. Saini R, Saini S, Sharma S (2011) Biofilm: A dental microbial infection. J Nat Sci Biol Med 2: 71-75.

10. Valen $H$, Scheie AA (2018) Biofilms and their properties. Eur J Oral Sci 126: 13-18.

11. Razak FA, Rahim ZHA (2013) Oral microbes and its environment: A review article. Esteem Academic Journal 9: 67-75.

12. Kriebel K, Hieke C, Hilke B M, Nakata M, Kreikemeyer B (2018) Oral biofilms from symbiotic to pathogenic interactions and associated disease-connection of periodontitis and rheumatic arthritis by peptidyl arginine deiminase. Front. Microbiol 9: 53.

13. Jiao Y, Tay FR, Niu L, Chen J (2019) Advancing antimicrobial strategies for managing oral biofilm infections. IJOS 11: 28. 
14. Word Health Organization (2020) Oral health.

15. Marsh P, Lewis M, Rogers H, Williams D, Wilson M (2016) Oral microbiology. (6 $6^{\text {th }}$ edn), Elsevier Ltd, Edinburgh, London, New York.

16. Grover V, Kapoor A (2018) Discriminating life forms in oral biofilms. Oral microbiology in periodontitis.

17. Szafranski SP, Winke A, Stiesch M (2017) The use of bacteriophages to biocontrol oral biofilms. J Biotechnol 250: 29-44.

18. Kuang X, Chen V, Xu X (2028) Novel approaches to the control of oral microbial biofilms. Biomed Res Int.

19. Bowen WH, Burne RA, Wu H, Koo H (2018) Oral biofilms: Pathogens, matrix and polymicrobial interactions in microenvironments. Trends Microbiol 26: 229-242.

20. Licker M, Moldovan R, Hogea E, Delia M, Florin H, et al. (2017) Microbial biofilm in human health-an updated theoretical and practical insight. Revista Română de Medicină de Laborator 25.

21. Gurenlian JAR (2007) The role of dental plaque biofilm in oral health. JDH 81.

22. Twetman S (2018) Prevention of dental caries as a non-communicable disease. Eur J Oral Sci 126: 19-25.

23. Koo H, Falsetta ML, Klein MI (2013) The exopolysaccharide matrix: $A$ virulence determinant of cariogenic biofilm. $J$ Dent Res 92: 1065-1073.

24. Gupta P, Gupta N, Singh HP (2014) Prevalence of dental caries in relation to body mass index, daily sugar intake, and oral hygiene status in 12-year-old school children in mathura city: A pilot study, Int J Pediatr 2014.

25. Lin NJ (2017) Biofilm over teeth and restorations: What do we need to know? Dent Mater 33: 667-680.

26. Donlan RM, Costerton WJ (2002) Biofilms: Survival mechanisms of clinically relevant microorganisms. Clin Microbiol Rev 15: 167-193.

27. Do T, Devine D, Marsh PD (2013) Oral biofilms: Molecular analysis, challenges, and future prospects in dental diagnostics. Clin Cosmet Investig Dent 5: 11-19.

28. Krzyściak W, Jurczak A, Piątkowski J (2016) The role of human oral microbiome in dental biofilm formation. Microbial biofilms-importance and applications.

29. Ayoub HM, Gregory RL, Tang Q, Lippert F (2020) Influence of salivary conditioning and sucrose concentration on biofilm-mediated enamel demineralization. J Appl Oral Sci 28.

30. Siqueira WL, Helmerhorst EJ, Zhang W, Salih E, Oppenheim FG (2007) Acquired enamel pellicle and its potential role in oral diagnostics. Ann N Y Acad Sci 1098: 504-509.

31. Hara AT, Zero DT (2010) The caries environment: Saliva, pellicle, diet, and hard tissue ultrastructure. Dent Clin North Am 54: 455-467.

32. Belibasakis GN, Bostanci N (2017) Oral biofilms and their implication in oral diseases, In: Seneriratne CJ, Microbial Biofilms. ( $1^{\text {st }}$ edn), Antimicrobials and clinical implications, CRC Press, Taylor and Francis Group, Boca Raton, London, New York.

33. Naumova EA, Weber L, Pankratz V, Czenskowski V, Arnold WH (2019) Bacterial viability in oral biofilm after tooth brushing with amine fluoride or sodium fluoride. Arch Oral Biol 97: 91-96.

34. Kressirer CA, Chen T, Harriman KL, Frias Lopez J, De- whirst FE, et al. (2018) Functional profiles of coronal and dentin caries in children. J Oral Microbiol 10.

35. Xu X, Chen F, Huang Z, Ma L, Chen L, et al. (2018) Meeting report: $A$ close look at oral biofilms and microbiomes. Int $J$ Oral Sci 10: 28.

36. Hu X, Huang YY, Wang Y, Wang X, Hamblin MR (2018) Antimicrobial photodynamic therapy to control clinically relevant biofilm infections. Front Microbiol 9: 1299.

37. Rezende G, Arthur RA, Lamers ML, Hashizume LN (2019) Structural organization of dental biofilm formed in situ in the presence of sucrose associated to maltodextrin. Braz Dent J 30: 36-42.

38. Matz C (2011) Competition, communication, cooperation: Molecular crosstalk in multi-species biofilms. In: Flemming HC, Wingender J, Szewzyk U, Biofilm Highlights. Springer, Berlin, Heidelberg.

39. Marsh PD, Head DA, Devine DA (2015) Dental plaque as a biofilm and a microbial community-implications for treatment. JOB 57: 185-191.

40. Marsh PD (2004) Dental plaque as a microbial biofilm. Caries Res 38: 204-211.

41. Rode Sde M, Gimenez X, Montoya VC, Gómez M, Blanc SL, et al. (2012) Daily biofilm control and oral health: Consensus on the epidemiological challenge--Latin American Advisory Panel. Braz Oral Res 26: 133-143.

42. Marsh PD, Devine DA (2011) How is the development of dental biofilms influenced by the host? J Clin Periodontol 38: 28-35.

43. Liu Y (2019) Dextran-coated iron oxide nanoparticles as biomimetic catalysts for biofilm disruption and caries prevention. Dental Theses.

44. Marsh PD (2006) Dental plaque as a biofilm and a microbial community-implications for health and disease, BMC Oral Health 6: S14.

45. Paes Leme AF, Koo H, Bellato CM, Bedi G, Cury JA (2006) The role of sucrose in cariogenic dental biofilm formation-new insight. J Dent Res 85: 878-887.

46. Tinanoff N, Palmer CA (1993) Dietary determinants of dental caries and dietary recommendations for preschool children. J Public Health Dent 60: 197-206.

47. Hujoel PP, Lingström $P$ (2017) Nutrition, dental caries and periodontal disease: A narrative review. J Clin Periodontol 44: S79-S84.

48. Jahangiry L, Bagheri R, Darabi F, Sarbakhsh P, Sistani MMN, et al. (2020) Oral health status and associated lifestyle behaviors in a sample of Iranian adults: An exploratory household survey. BMC Oral Health 20: 82.

49. Akbar T, Hasan H, Awal A, Ghosh MK, Haque J, et al. (2017) A study on biofilm formation in relation to oral hygiene status related to tooth brushing practice. Up DCJ 7.

50. Hitz Lindenmüller I, Lambrecht JT (2011) Oral care. Curr Probl Dermatol 40: 107-115.

51. Qin M, Li J, Zhang S, Ma W (2008) Risk factors for severe early childhood caries in children younger than 4 years old in Beijing, China. Pediatr Dent 30: 122-128.

52. Wulaerhan J, Abudureyimu A, Bao XL, Zhao J (2014) Risk determinants associated with early childhood caries in Uygur children: A preschool-based cross-sectional study. BMC Oral Health 14: 136.

53. Kayombo CM, Mumghamba EG (2017) Self-reported hali- 
tosis in relation to oral hygiene practices, oral health status, general health problems, and multifactorial characteristics among workers in ilala and temeke municipals, Tanzania. Int J Dent 2017: 8682010.

54. Zijnge V, Van Leeuwen MBM, Degener JE, Abbas F, Thurnheer T, et al. (2010) Oral biofilm architecture on natural teeth. PLoS One 5: e9321.

55. Zhu B, Macleod LC, Kitten T, Xu P (2018) Streptococcus sanguinis biofilm formation \& interaction with oral pathogens. Future Microbiol 13: 915-932.

56. Sheiham A (2005) Oral health, general health and quality of life. Bull World Health Organ 83: 644.

57. Mariano S, David B, Michael AC, Jaime AC, Irene Dige, et al. (2017) Role of microbial biofilms in the maintenance of oral health and in the development of dental caries and periodontal diseases. Consensus report of group 1 of the joint EFP/ORCA workshop on the boundaries between caries and periodontal disease. J Clin Periodontol 44: S5-S11.

58. Peyyala R, Kirakodu SS, Novak KF, Ebersole JL (2012) Oral microbial biofilm stimulation of epithelial cell responses. Cytokine 58: 65-72.

59. Struzycka I (2014) The oral microbiome in dental caries. Pol J Microbiol 63: 127-135.

60. Petersen PE, Lennon MA (2004) Effective use of fluorides for the prevention of dental caries in the $21^{\text {st }}$ century: The WHO approach. Community Dent Oral Epidemiol 32: 319321.

61. Mathur VP, Dhillon JK (2018) Dental caries: A disease which needs attention. Indian J Pediatr 85: 202-206.

62. Cirino SM, Scantlebury S (1998) Dental caries in developing countries. Preventive and restorative approaches to treatment. N Y State Dent J 64: 32-39.
63. Miura H, Araki Y, Haraguchi K, Arai Y, Umenai T (1997) Socioeconomic factors and dental caries in developing countries: A cross-national study. Soc Sci Med 44: 269-272.

64. Pitts NB, Zero DT, Marsh PD, Kim E, Jane AW, et al. (2017) Dental caries. Nat Rev Dis Primers 3: 17030.

65. Tafere Y, Chanie S, Dessie T, Gedamu H (2018) Assessment of prevalence of dental caries and the associated factors among patients attending dental clinic in Debre Tabor general hospital: A hospital-based cross-sectional study. BMC Oral Health 18: 119.

66. Seid E, Derseh L, Derso T, Assefa M, Gonete KA, et al. (2018) Nutrient consumption and associated factors among school age children in Dewa Chefe District, northeast Ethiopia: A cross-sectional study. BMC Res Notes 11: 669.

67. Bicak DA (2018) A current approach to halitosis and oral malodor- a mini review. Open Dent J 12: 322-330.

68. Kolo ES, Idemudia AB, Ahmed AO, Alufohai O (2015) Psychosocial problems in adults with halitosis, JMT 17: 87-90.

69. Gonçalves ACS, Martins MCN, Paula BL, Weckwerth PH, Franzolin SOB, et al. (2019) A new technique for tongue brushing and halitosis reduction: The $X$ technique. $J$ Appl Oral Sci 27: e20180331.

70. Quadri MFA, Shubayr MA, Hattan AH, Wafi SA, Jafer AH (2018) Oral hygiene practices among Saudi Arabian children and its relation to their dental caries status. Int J Dent.

71. Santos A (2003) Evidence-based control of plaque and gingivitis. J Clin Periodontol 30: 13-16.

72. Tao D, Li F, Feng X, Wong MCM, Lu H (2018) Plaque biofilm microbial diversity in infants aged 12 months and their mothers with or without dental caries: A pilot study. BMC Oral Health 18: 228. 